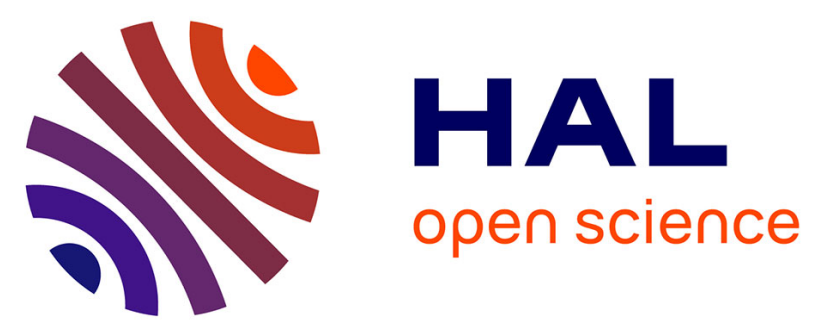

\title{
Three-dimensional measurement of Mg dopant distribution and electrical activity in GaN by correlative atom probe tomography and off-axis electron holography
}

Lynda Amichi, Isabelle Mouton, Enrico Di Russo, Victor Boureau, Frédéric Barbier, Amélie Dussaigne, Adeline Grenier, Pierre-Henri Jouneau, Catherine Bougerol, David Cooper

\section{To cite this version:}

Lynda Amichi, Isabelle Mouton, Enrico Di Russo, Victor Boureau, Frédéric Barbier, et al.. Threedimensional measurement of $\mathrm{Mg}$ dopant distribution and electrical activity in GaN by correlative atom probe tomography and off-axis electron holography. Journal of Applied Physics, 2020, 127 (6), pp.065702. 10.1063/1.5125188 . hal-02991526

\section{HAL Id: hal-02991526 \\ https://hal.science/hal-02991526}

Submitted on 19 Nov 2020

HAL is a multi-disciplinary open access archive for the deposit and dissemination of scientific research documents, whether they are published or not. The documents may come from teaching and research institutions in France or abroad, or from public or private research centers.
L'archive ouverte pluridisciplinaire HAL, est destinée au dépôt et à la diffusion de documents scientifiques de niveau recherche, publiés ou non, émanant des établissements d'enseignement et de recherche français ou étrangers, des laboratoires publics ou privés. 


\title{
Three-dimensional measurement of $\mathrm{Mg}$ dopant distribution and electrical activity in GaN by correlative atom probe tomography and off-axis electron holography.
}

\author{
Lynda Amichi' ${ }^{1}$, Isabelle Mouton ${ }^{2}$, Enrico Di Russo², Victor Boureau², Frédéric Barbier ${ }^{2}$, Amélie \\ Dussaigne $^{2}$, Adeline Grenier ${ }^{2}$, Pierre-Henri Jouneau ${ }^{1}$, Catherine Bougerol ${ }^{1,3}$ and David Cooper ${ }^{2}$ \\ ${ }^{1}$ Université Grenoble Alpes, CEA, INAC, F-38000 Grenoble, France. \\ ${ }^{2}$ Université Grenoble Alpes, CEA, LETI, F-38000 Grenoble, France. \\ ${ }^{3}$ Université Grenoble Alpes, CNRS, Institut Néel, F-38000 Grenoble, France.
}

Corresponding author david.cooper@cea.fr

The distribution and electrical activity of p-type doping $(\mathrm{Mg})$ in gallium nitride $(\mathrm{GaN})$ grown by metal organic chemical vapor deposition was investigated by correlating atom probe tomography (APT) and off-axis electron holography. APT results revealed that high $\mathrm{Mg}$ concentrations promote the formation of $\mathrm{Mg}$-rich clusters. This is associated with the formation of pyramidal inversion domains (PIDs). The direct measurement of the doping concentration outside the clusters provided by APT suggests a saturation in the p-type electrical activity for $\mathrm{Mg}$ concentrations above $7 \times 10^{19} \mathrm{~cm}^{-3}$. Maps of the electrostatic potential provided by off-axis electron holography confirm that the highest carrier concentration was achieved in the regions with the highest dopant concentration of $2 \times 10^{20} \mathrm{~cm}^{-3}$, despite the presence of a high density of $\mathrm{Mg}$ rich clusters revealed by APT. The correlation of these techniques suggests that PIDs are not the major cause of the reduction in electrostatic potential.

Introduction

GaN and its alloys are of a great interest in the fabrication of high-performance devices that are widely used in optoelectronic applications as well as power electronics. Despite their widespread use, the ability to produce $p$ type doping is still a challenge [1]. Mg is currently the only efficient p-type dopant in GaN, but due to its high ionization energy [2], high dopant concentrations of up to $1 \times 10^{20} \mathrm{~cm}^{-3}$ are needed to achieve the required hole concentration at room temperature [3]. An important drop in the carrier concentration has been observed above $\mathrm{Mg}$ concentrations in the low $10^{19} \mathrm{~cm}^{-3}$ range [4]. This is thought to be due to combinations of at least two defect mechanisms: (i) the formation of Pyramidal Inversion Domains (PIDs), containing electrically inactive $\mathrm{Mg}$ atoms [5-7]; (ii) the formation of complexes with native defects such as $M g V_{N}$, where $V_{N}$ is a nitrogen vacancy [8-10]. To better understand the influence of the PIDs on the reduction of the Mg dopant activity, correlative Atom Probe Tomography (APT) and off-axis electron holography were performed. APT provides a near atomic-scale 3D image of the $\mathrm{Mg}$ doping distribution [11,12] which is correlated with the $\mathrm{nm}$-scale measurement of the electrical activity of active dopants by off-axis electron holography $[13,14]$.

Experimental Results

A wafer containing differently doped layers was grown along the [0001] direction by metal organic chemical vapor deposition (MOCVD) at $950{ }^{\circ} \mathrm{C}$ on a n-type doped-GaN substrate. The conducting substrate reduces the presence of dislocations and the charging effects during holography measurements. The sample consists of three successive GaN layers with increasing $\mathrm{Mg}$ dopant concentrations being $400 \mathrm{~nm}$ of $3 \times 10^{19} \mathrm{~cm}^{-3}, 300 \mathrm{~nm}$ of $7 \times 10^{19} \mathrm{~cm}^{-3}$ and then $200 \mathrm{~nm}$ of $2 \times 10^{20} \mathrm{~cm}^{-3}$. Finally, a $500 \mathrm{~nm}$ thick Not Intentionally Doped (NID) capping 
layer was grown. The sample was intentionally designed to have different dopant concentrations in the same stack. This is due to the difficulty in comparing small changes in electrostatic potential when examining different TEM specimens separately by off-axis electron holography. Figure 1(a) shows a Secondary lon Mass Spectrometry (SIMS) profile revealing the total Mg concentrations in the differently doped layers with their respective thicknesses. This profile also reveals an unintended $\mathrm{Mg}$ concentration of $1.5 \times 10^{19} \mathrm{~cm}^{-3}$ in the first $50 \mathrm{~nm}$ of the NID layer. This effect is due to the surfactant properties of the Mg dopants, which remain on the surface during growth process and are incorporated in the NID layer grown above.

A TEM lamella and needle-shaped atom probe specimens were prepared using a standard lift-out procedure using a Dual-Beam Focused Ion Beam (FIB) operated at $16 \mathrm{kV}$ with a final cleaning step at $2 \mathrm{kV}$. High-Angle Annular Dark Field Scanning Transmission Electron Microscopy (HAADF-STEM) was performed on a thin $<100$ $\mathrm{nm}$ ) lamella and observed down the [11-20] direction using an aberration corrected FEI Titan Ultimate operated at $300 \mathrm{kV}$. A low magnification image is shown in figure 1(b). The position and thickness of the doped layers are revealed from the bright contrast. Higher magnification HAADF-STEM images of the highly doped layers with the nominal $\mathrm{Mg}$ concentrations of $2 \times 10^{20}$ and $7 \times 10^{19} \mathrm{~cm}^{-3}$ are also shown in figures 1 (c) and (d) respectively. The bright contrast regions, corresponding to the presence of PIDs, reveals the highest density for the highest $\mathrm{Mg}$ concentration of $2 \times 10^{20} \mathrm{~cm}^{-3}$. The triangular shape of such PIDs can be better observed at a concentration of $7 \times 10^{19} \mathrm{~cm}^{-3}$, where they are more dispersed. In these HAADF-STEM images no PIDs are observed in the lowest doped layers $\left(\leq 3 \times 10^{19}\right)$.

Laser-assisted Atom Probe Tomography (La-APT) was performed using a CAMECA FlexTAP operated in UV mode. The analysis parameters included an operating temperature of $T=40 \mathrm{~K}$, a peak energy density during the laser pulse of $2.7 \times 10^{-4} \mathrm{~J} \mathrm{~cm}^{-2}$ and a detection rate of 0.01 event/pulse. The FlexTAP detection efficiency was $62 \%$, corresponding to the active area of the multi-channel plate [15]. The APT experiment was performed under low electric field conditions, as suggested by the low charge-to-state ratio Ga2+/Ga+ resulting during the tip evaporation. This ratio was approximately constant during the whole experiment it and ranged between 0.021 and 0.025 , corresponding to a surface field of about $23 \mathrm{~V} \mathrm{~nm}^{-1}$ [16]. Despite these conditions, the evaporation of the GaN layer with a dopant concentration of $3 \times 10^{19} \mathrm{~cm}^{-3}$, which is deep in the structure, was never observed because of systematic tip ruptures. In such low field conditions, an overestimation of the metallic elements, Mg and Ga is expected, leading to a deficiency in the N measurement $[16,17]$. Therefore, the cation III-site metallic fraction should approach the expected value and can be considered for an estimation of the $\mathrm{Mg}$ composition in GaN in the different regions [18]. However, as long as the evaporation conditions remain constant, such as the electric field at the apex, the relative comparison of the Mg properties between the several layers is reliable.

A schematic of the region that was examined by APT is represented with red dashes in the HAADF-STEM image of the lamella specimen shown in figure 2(a). The distribution of $\mathrm{Mg}$ atoms within the 3D reconstructed volume is revealed in figure 2(b). Three regions corresponding to the three $\mathrm{Mg}$ doped layers can be observed. The $\mathrm{Mg}$ composition profile calculated from the reconstructed volume and expressed in terms of $\mathrm{Mg}$ metallic fraction, $y$ $=\mathrm{Mg} /(\mathrm{Mg}+\mathrm{Ga})[16,17]$ is shown in figure 2(c). This composition profile allows the three differently doped regions to be distinguished. Region (1), which is the NID layer, has an average value of $y=0.15 \pm 0.04 \%$. The extracted mass spectrum is shown in Figure 2(d) where only the more abundant isotope ${ }^{24} \mathrm{Mg}^{2+}$ is detected, indicating that this measurement is close to the detection limits of APT (about $1 \times 10^{19} \mathrm{~cm}^{-3}$ ) [18]. Region (2) corresponds to the most highly doped layer at $2 \times 10^{20} \mathrm{~cm}^{-3}$, where a value of $y=0.40 \pm 0.01 \%$ was measured. Figure 2(b) shows the presence of a high density of $\mathrm{Mg}$ clustering in this layer. Region (3) is the layer doped at 7 $x 10^{19} \mathrm{~cm}^{-3}$, where $y=0.30 \pm 0.02 \%$ is obtained. In this layer, the APT suggests less Mg clustering than for the highest doped layer, confirmed by the HAADF-STEM observations. As previously discussed, region (4) with a Mg concentration of $3 \times 10^{19} \mathrm{~cm}^{-3}$ was not examined in this study due to numerous tip breakages during the long data acquisition times.

A cluster identification procedure was performed in the APT 3D reconstructed volume. This consists of a maximum separation technique based on the calculation of distance between first nearest neighbour of solute atoms $[19,20]$. Then from this identification, the number of $\mathrm{Mg}$ atoms can be determined inside each cluster as well as the overall cluster density inside each p-doped layer. Using this methodology an increase in the cluster 
density from $1.2 \times 10^{17} \mathrm{~cm}^{-3}$ to $1.8 \times 10^{18} \mathrm{~cm}^{-3}$ was observed where the total $\mathrm{Mg}$ concentration measured by SIMS increases from $7 \times 10^{19}$ to $2 \times 10^{20} \mathrm{~cm}^{-3}$ respectively. These observations are in qualitative agreement with the HAADF-STEM observation shown in Figure 1. One of the key strengths of APT is not only to be able to visualize the Mg-rich clusters, but also to calculate the remaining $\mathrm{Mg}$ outside of the clusters which are considered to be potentially electrically active (non-PID). Figure 2(e) shows both the total metallic fraction measured by APT, and the non-PID fraction for the different dopant concentrations in the regions examined. When considering the total $\mathrm{Mg}$ concentration, the metallic fraction increases with the total $\mathrm{Mg}$ concentration as expected. However, after removing the atoms contained in the clusters, there is an increase in the non-PID $\mathrm{Mg}$ concentration when comparing region (1) (total concentration $1.5 \times 10^{19} \mathrm{~cm}^{-3}$ ) to region (3) (total concentration $7 \times 10^{19} \mathrm{~cm}^{-3}$ ) despite the formation of the $\mathrm{Mg}$-rich clusters for higher dopant concentrations. The measured increase of the non-PID fraction across these layers is from $0.15+/-0.04 \%$ to $0.29+/-0.01 \%$ respectively. For region (2) $\left(2 \times 20 \mathrm{~cm}^{-3}\right)$ the measured value of non-PID Mg concentration is $0.27+/-0.015 \%$, thus despite the very high-density of the Mg-rich clusters, the dopant concentration that is potentially active remains constant to within error. To provide more information about the activity of the dopants, off-axis electron holography was performed to measure the relative changes in electrostatic potential across the layers.

Off-axis electron holography is a TEM based technique that uses an electron biprism to interfere a coherent electron wave that passes through a region of interest with another that passes through vacuum. This interference pattern, also known as hologram, contains information about the amplitude and the phase of the electrons [13]. In the absence of dynamical diffraction effects and magnetic fields, the change in phase is directly proportional to the Mean Inner Potential of the material ( $\left.V_{M I P}\right)$ and variations of the electrostatic potential arising from the presence of active dopants $\left(V_{\text {dopant }}\right)$. The measured change in phase is given by:

$$
\Delta \Phi=C_{E} \int_{0}^{t}\left(V_{\text {dopant }}+V_{M I P}\right) d z,
$$

where $t$ is the thickness of the specimen; $C_{E}$ is a constant equal to $0.0073 \mathrm{rad} \cdot \mathrm{nm}^{-1} \cdot \mathrm{V}^{-1}$ for $200 \mathrm{kV}$ electrons; the $V_{\text {MIP }}$ has been calculated to be $16.8 \mathrm{~V}$ for GaN from density functional theory [21]. A parallel-sided and flat lamella specimen is required in order to accurately measure the step in phase coming from $V_{\text {dopant. }}$ Under these conditions, the change in phase can then be described as it follows:

$$
\Delta \Phi=C_{E} V_{\text {dopant }}\left(t_{\text {cryst }}-t_{\text {inactive }}\right) \text {, }
$$

where $t_{\text {cryst }}$ is the crystalline thickness of the specimen. The inactive thickness, $t_{\text {inactive }}$ is a crystalline surface region which does not contribute towards the measured phase change arising from the active dopants. This is caused by a combination of structural damage caused by FIB milling, which can trap the dopant atoms in defect states; and the effect of surface charging, or pinning, which leads to band bending at the specimen surfaces [22-23]. It is known that a step in potential which is much less than expected from theory has been measured in doped GaN p-n junctions [24]. Therefore, to maximize the measured step in phase a $500 \pm 10 \mathrm{~nm}$ thick lamella was examined. The crystalline thickness was measured by using 2-beam convergent beam electron diffraction (CBED) and comparing the results to simulations. To reduce the effects of specimen charging, a low electron beam current of $3 \mathrm{e}^{-} \AA^{-2} \mathrm{~s}^{-1}$ was used while the signal-to-noise ratio was improved by the summation of a series of 32 holograms using the Holoview software [25]. Finally, as the Mg dopant is a deep acceptor with an ionization energy in the range $100-200 \mathrm{meV}$ depending on the concentration [2], the ionization rate is low at room temperature. As a consequence, in order to increase the carrier concentration and reduce the artifacts associated with the electrically inactive thickness [26], the specimen was examined in-situ at a temperature of $400{ }^{\circ} \mathrm{C}$ using a Gatan heating holder. According to the charge neutrality equation [2,3], for the concentration of $1 \times 10^{19} \mathrm{~cm}^{-3}$ and $2 \times 10^{20} \mathrm{~cm}^{-3}$ the theoretical ionization rate reaches $15 \%$ and $24 \%$ at $400{ }^{\circ} \mathrm{C}$, compared to $2.2 \%$ and $4.5 \%$ at room temperature respectively. Higher annealing temperatures were not used to ensure that there were no problems with dopant diffusion in the specimens.

The off-axis electron holography experiments were performed using a FEI Titan Themis TEM operated at $200 \mathrm{kV}$. A biprism voltage of $150 \mathrm{~V}$ was used to provide a hologram width of about $700 \mathrm{~nm}$ and a fringe spacing of $3 \mathrm{~nm}$. To reduce the dynamical diffraction effects, the specimen was tilted at about $2^{\circ}$ from the zone axis while 
keeping the region of interest edge on. Series of holograms of the sample were acquired with a corresponding reference series of vacuum, each with an exposure time of 8 seconds using a 2048x2048 pixels CCD camera. The holograms were reconstructed and the complex images were aligned before reconstruction to provide phase images with a spatial resolution of $9 \mathrm{~nm}$. Much higher spatial resolutions can be achieved [25], but these experimental parameters were optimized to obtain a large field of view. The phase image acquired at $400{ }^{\circ} \mathrm{C}$ is shown in figure $3(\mathrm{a})$ where the contrast reveals the p-type doped layers at $2 \times 10^{20}, 7 \times 10^{19}$ and $3 \times 10^{19} \mathrm{~cm}^{-3}$. The NID region with a dopant concentration of $1.5 \times 10^{19} \mathrm{~cm}^{-3}$ can also be identified. Above and below the $\mathrm{Mg}$ doped layers, a bright contrast due to the residual n-type doping which is estimated to be $1 \times 10^{17} \mathrm{~cm}^{-3} \mathrm{can} \mathrm{be}$ observed [27]. Figure $3(\mathrm{~b})$ shows the $V_{\text {dopant }}$ profile that was extracted directly from the region indicated in figure 3(a). As the specimen was parallel-sided across the region of interest it was not necessary to perform any corrections for small changes in specimen thickness. A step in potential of $130+/-10 \mathrm{mV}$ is measured between the NID region (1) (dopant concentration of $\left.1.5 \times 10^{19}\right)$ and the region $(2)\left(2 \times 10^{20}\right) \mathrm{cm}^{-3}$. A second step in potential of $50 \pm 10 \mathrm{mV}$ is measured between the region (2) $\left(2 \times 10^{20} \mathrm{~cm}^{-3}\right)$ and region (3) $\left(7 \times 10^{19} \mathrm{~cm}^{-3}\right)$. The $V_{\text {dopant }}$ profile in figure $3(\mathrm{~b})$ shows that the layer with a dopant concentration of $2 \times 10^{20} \mathrm{~cm}^{-3}$ has the lowest potential. This result suggests that the $p$-type carrier concentration in this region is highest despite having the highest density of PIDs. Thus, since the p-type activity is improved despite the large increase in the Mg-rich clusters density, we can conclude qualitatively at this point the presence of the PIDs do not cause the drop in carrier concentration in these specimens.

To obtain a more quantitative measurement of the active dopants in the layers, the 2D electrostatic potential in the $500 \mathrm{~nm}$ thick TEM lamella was simulated using the Nextnano ${ }^{3}$ software [28]. As well as the differently doped layers with their corresponding thicknesses, a residual n-type activity with an electron concentration of $1 \times 10^{17} \mathrm{~cm}^{-3}$ was used in the whole structure [27]. Moreover, the presence of charged surfaces resulting from the presence of damaged surface layers and their interaction with the electron beam was included. These modified surfaces introduce energy levels which cause band bending at the interface with vacuum and pin the Fermi level mid gap at $1.7 \mathrm{eV}$ [22]. An unexpected advantage of FIB preparation is that the Ga implanted surfaces of the specimen provide good conduction paths, such that the the build up of charge in the specimens is reduced and dynamic charging effects or fringing fields are rarely observed during semiconductor device studies. However the implantation of Ga ions during FIB preparation also creates a further damaged area below the amorphous surfaces of the specimen which remain crystalline but where the dopants are electrically inactive [20]. The inactive thickness was measured experimentally to be $40 \mathrm{~nm}$ on each side of the specimen using a methodology described elsewhere [29]. Due to compensation effects and PIDs, the active Mg dopant concentration is smaller than measured by SIMS. Although the non-PID Mg concentration has been estimated, the compensation rate is unknown in this case. As a starting point, a value of $50 \%$ of compensation was used for the highest-doped layer. The active concentration was then adapted in regions (1) and (3) then the values were iterated in order to reproduce the experimentally measured step in potential. Finally, the ionization energy dependence with the active concentration in each layer was considered [2]. The 2D potential distribution in the simulated structure obtained for the best fitting active Mg dopant concentrations of $8 \times 10^{18}$, $6 \times 10^{19}$ and $3 \times 10^{19} \mathrm{~cm}^{-3}$ is shown in figure $3(\mathrm{c})$. The overall inactive thickness $\left(t_{\text {inactive }}\right)$ coming from both structural damage and band bending can be observed in the simulation. The projected potential profile through the thickness of the specimen obtained from simulation is shown in figure 3(b). The different potential levels in the regions and the depletion region toward NID layers corresponding to an intrinsic-p junction can be observed. In the doped layers (1), (2) and (3) the simulated profile is in agreement with the experimental profile. However, discrepancies can be observed between simulations and experiment at the top surface and the substrate regions. This match could be improved by including the variations of intrinsic dopants and also by considering more accurately the effects of FIB damage in the top part of the specimen.

\section{Discussion}

Table 1 shows the total Mg concentrations measured by SIMS and the non-PID Mg measured by APT. As it is not possible to directly quantify the APT results at this time, a quantitative value for the non-PID Mg has been obtained by multiplying the SIMS measurements by the ratio of the total metallic fraction and the non-PID Mg fraction shown in Figure 2(e). The APT measurement of the non-PID Mg dopants alone would suggest that the $\mathrm{p}$-type electrical activity would increase from a nominal $\mathrm{Mg}$ concentration of $1.5 \times 10^{19} \mathrm{~cm}^{-3}$ to $7 \times 10^{19} \mathrm{~cm}^{-3}$ and 
then plateau at the concentration of $2 \times 20 \mathrm{~cm}^{-3}$. However, by quantifying them with SIMS it is possible to obtain the highest value of potentially active dopants $\left(1.34 \times 20 \mathrm{~cm}^{-3}\right)$ in the region with the highest dopant concentration. These measurements however do not account for the presence of complexes and native defects, thus at this stage it is not possible to determine whether the dopants outside of the clusters are indeed electrically active. The electron holography reveals that the highest active dopant concentration is still in the highest doped layer. Further correlation of electron holography with simulations show that it is possible to obtain an insight into the concentrations of non-PID dopants that are rendered inactive due the presence of complexes such as from the $\mathrm{MgV}_{\mathrm{N}}$ vacancy. The values shown in Table 1 comparing the non-PID and active dopants suggest that for all of the different dopant concentrations examined here, approximately half of the non-PID Mg atoms are active. These measurements suggest that for these samples, the layer with the highest dopant concentration has an active concentration of $0.6 \times 10^{20} \mathrm{~cm}^{-3}$ out of a total $\mathrm{Mg}$ concentration of $2 \times 10^{20}$ $\mathrm{cm}^{-3}$ despite having the highest concentration of PIDS. For better visualization of the experimental results, Figure 4 shows a summary of the non-PID and active dopants plotted as a function of the total dopant concentration measured by SIMS.

The possibility that the experimental conditions used in TEM and specimen preparation can also influence the measurements of the electrical potential should also be considered. For example in preparing a 500-nm-thick TEM lamella, the hydrogen which is known to passivate $\mathrm{Mg}$ dopants could escape from the specimen and provide misleading results [31]. However, annealing temperatures used from driving the hydrogen laterally from GaN devices such as tunnel junctions are typically higher than $700^{\circ} \mathrm{C}[31,32]$. This is much higher than the temperatures expected during either FIB milling or during the in-situ annealing experiments. Additionally, it is known that local heating during caused by $10 \mathrm{kV}$ electrons during scanning electron microscopy (SEM) observations can activate the dopants [33,34]. In the case of $200 \mathrm{kV}$ electrons, significant heating is not expected and knock-on beam damage would be the major interaction with the specimen which would lead to a decrease in measured dopant activity. In similar experiments, the TEM specimen was held at $400^{\circ} \mathrm{C}$ for several hours under electron beam irradiation to assess the stability of the Mg-doped GaN and no changes in the electrical activity was observed [35]. In addition, no changes in potential were observed after the specimen in this work was examined at room temperature, annealed at $400^{\circ} \mathrm{C}$ for two hours and then re-examined at room temperature. For future experiments, further correlation with electrical measurements such as Hall probe should be performed to assess the effects of specimen preparation on the measurements made by electron holography. Ideally, these studies would be performed on separate specimens each containing different a dopant concentration with the carrier concentrations measured electrically. However, even for laboratories that are experienced in performing electron holography, the difficulties of specimen preparation and performing the individual experiments would make conclusive measurements extremely complicated. Even during careful experimental control, it would be difficult to be sure that the very small differences in potential $(50 \mathrm{mV}$ ) were only from dopant activity and not from parameters such as Ga ion damage, dynamical diffraction in the thick TEM specimens, errors in the measurement of the specimen thickness and charging under the electron beam. As such, at this time it is preferable to measure stacks of differently doped material by electron holography such that the experimental conditions can be controlled across the whole region of interest.

Previous studies that are reported in literature show that the carrier concentrations measured by the Hall effect can decrease strongly after a Mg concentration threshold of about $3 \times 10^{19} \mathrm{~cm}^{-3}$ for MOCVD or HVPE grown structures $[5,30]$. Indeed, an efficient p-type activity is generally difficult to achieve at high dopant concentrations using high growth-temperature techniques. However, these experimental results are consistent with previous studies where the reduction of the carriers did not occur up to $\mathrm{Mg}$ dopant concentrations of more than $2 \times 10^{20} \mathrm{~cm}^{-3}$ [3], although this work did not link the measured dopant activity to the presence of PIDs. Thus when comparing the measurements presented in this work and those in the bulk of the literature we conclude that the active carrier concentration in $\mathrm{GaN}$ is very dependent on the growth conditions.

\section{Conclusion}

The correlation between APT and electron holography has allowed the direct link between the nm-scale spatial distribution and the electrical activity of $\mathrm{Mg}$ dopants in our GaN nanostructure as a function of the total concentration that is incorporated during growth. The APT measurements have shown that highest densities of 
clusters that are associated to the presence of PIDs, appear at the highest Mg doping concentrations. This observation is in agreement with HAADF-STEM investigations. These clusters contain Mg dopants which are electrically inactive and reduce the concentration of potentially active $\mathrm{Mg}$. The APT alone would suggest that the highest concentration of $\mathrm{Mg}$ that is potentially active is observed in the region with a concentration of $7 \mathrm{x}$ $10^{19} \mathrm{~cm}^{-3}$. The APT has been combined with SIMS to provide a quantitative measure of the dopants that are outside of the PIDs and are therefore potentially active.

The methodology to perform electron holography has been adapted by using thick specimens, data averaging, low beam currents and in-situ annealing to improve both the signal-to-noise ratio and accuracy of the measurement. The use of electron holography has revealed that the dopant concentration of $2 \times 10^{20} \mathrm{~cm}^{-3}$ has the most efficient p-type activity despite it having the highest density of Mg-rich clusters. Although here we have reported the measurement of $\mathrm{Mg}$ doping in $\mathrm{GaN}$, these methods are widely transferrable to all doped semiconductor specimens. The ability to correlate these advanced characterisation techniques to obtain insights into the mechanisms leading to dopant inactivity will be an important tool in the development and improvement of new materials that are used for a wide range of applications.

\section{Acknowledgments}

Lynda Amichi thanks the GaNex organisation (ANR-11-LABEX-0014) for funding of her Phd position. GaNex belongs to the public funded "Investissements d'avenir" program managed by the French ANR Agency. David Cooper and Victor Boureau thank the European Research Council for the starting grant Holoview (Stg:306535). These experiments were performed on the Nano-Characterisation Platform (PFNC) at Minatec.

[1] T. Nakamura, S. Mukai, M. Senoh, and N. Iwasa, "Thermal annealing effects on p-type Mg-doped GaN films" Japanese J. Appl. Phys. 1992 31, 139.

[2] S. Brochen, J. Brault, S. Chenot, A. Dussaigne, M. Leroux, and B. Damilano, "Dependence of the Mgrelated acceptor ionization energy with the acceptor concentration in p-type GaN layers grown by molecular beam epitaxy" Applied Physics Letters, 2013 103, 032102.

[3] P. Kozodoy et al., "Heavy doping effects in Mg-doped GaN," Journal of Applied Physics, 200087, 1832-1835.

[4] M. Leroux et al. "Structural defects and Relation with optoelectronic Properties in highly MgDoped GaN" Physica Status Solidi (a), 2002192 394-400.

[5] P. Vennéguès et al., "Pyramidal defects in metalorganic vapor phase epitaxial Mg doped GaN" Aplp. Phys. Lett., 2000 77, 880-882.

[6] M. Leroux et al., "Pyramidal defects in highly Mg-doped GaN: atomic structure and influence on optoelectronic properties" The European Physical Journal : Applied Physics, 2004 27, 259-262

[7] S. Bennett et al., "Atom probe tomography and transmission electron microscopy of a Mg-doped AlGaN/GaN superlattice" Ultramicroscopy, 2011 111, 207-211.

[8] J. Neugebauer and C. G. Van de Walle, "Native defects and impurities in GaN" Advances in Solid State Physics 1996 35, 25-44

[9] P. Boguslawski, E. Briggs, and J. Bernholc, "Native defects in gallium nitride” Phys. Rev. B, 1995 51, 17255.

[10] T. Remmele, M. Albrecht, K. Irmscher, R. Fornari, and M. Strassburg, "Pyramidal inversion domain boundaries revisited" Appl. Phys. Lett., 2011 99, 141913.

[11] D. Blavette, A. Bostel, J. Sarrau, B. Deconihout, and A. Menand, "An atom probe for threedimensional tomography" Nature, 1993 363, 432.

[12] B. Gault, M. Moody, J. Cairney, and S. Ringer, Atom probe microscopy, vol. 160. Springer Science, 2012.

[13] A. Tonomura, "Applications of electron holography" Rev. Mod. Phys. 1987 59, 639

[14] W. D. Rau, P. Schwander, F. H. Baumann, W. Höppner, and A. Ourmazd, "Mapping of the Electrostatic Potential in Transistors by Electron Holography" Phys. Rev. Lett. 1999 82, 2614.

[15] M. Kelly "Atom probe tomography" Rev. Sci. Instruments 2007 78, 031101 
[16] E. Di Russo, I. Blum, J. Houard, M. Gilbert, G. Da Costa, D. Blavette, L. Rigutti, “Compositional Accuracy of Atom Probe Tomography Measurements in GaN: Impact of Experimental Parameters and Multiple Evaporation Events" Ultramicroscopy, 2018 187, 126-134.

[17] E. Di Russo, L. Mancini, F. Moyon, S. Moldovan, J. Houard, F. H. Julien, M. Tchernycheva, J. M. Chauveau, M. Hugues, G. Da Costa, I. Blum, W. Lefebvre, D. Blavette and L. Rigutti. "Three-dimensional atomic-scale investigation of $\mathrm{ZnO}-\mathrm{Mg}_{\mathrm{x}} \mathrm{Zn}_{1-\mathrm{x}} \mathrm{O}$ m-plane heterostructures." Appl. Phys. Lett., 2017111 032108.

[18] D. Diercks, B. Gorman, Kirchhofer., N. Sanford, K. Bertness, and M. Brubaker, “Atom probe tomography evaporation behavior of $\mathrm{C}$-axis GaN nanowires: Crystallographic, stoichiometric, and detection efficiency aspects" J. Appl. Phys., 2013 114, 184903.

[19] T. Philippe, M. Gruber, F. Vurpillot, D. Blavette, "Clustering and local magnification effects in atom probe tomography: A statistical approach" Microscopy and Microanalysis 2010, 16, 643-648.

[20) B. Gault, M. P. Moody, J. M. Cairney, S. P. Ringer, "Atom probe microscopy", Springer Science \& Business Media, 2012; Vol. 160.

[21] P. Kruse, M. Schowalter, D. Lamoen, A. Rosenauer, and D. Gerthsen, "Determination of the mean inner potential in III-V semiconductors, Si and Ge by density functional theory and electron holography" Ultramicroscopy, 2006106 105-113.

[22] P. Somodi, A. Twitchett-Harrison, P. Midgley, B. Kardynal, C. Barnes, and R. Dunin-Borkowski, "Finite element simulations of electrostatic dopant potentials in thin semiconductor specimens for electron holography" Ultramicroscopy, 2013 134, 160-166.

[23] D. Cooper, J-P. Barnes, J-M. Hartmann, P. Salles, G. Benassayag, R.E. Dunin-Borkowski “Dopant profiling of focused ion beam milled semiconductors using off-axis electron holography; reducing artifacts, extending detection limits and reducing the effects of gallium implantation" Ultramicroscopy, 2010 110, 383-389.

[24] J. Park, T. Niermann, D. Berger, A. Knauer, I. Koslow, M. Weyers, M. Kneissl and M. Lehmann, Impact of electron irradiation on electron holographic potentiometry" Appl. Phys. Lett., 2014 105, 094102.

[25] V. Boureau, R. McLeod, B. Mayall, and D. Cooper, "Off-axis electron holography combining summation of hologram series with double-exposure phase-shifting: theory and application" Ultramicroscopy, 2018 193, 52-63.

[26] D. Cooper, C. Ailliot, R. Truche, J-P. Barnes, J-M. Hartmann and F. Bertin “Experimental off-axis electron holography of focused ion beam-prepared Si pn junctions with different dopant concentrations." J. Appl. Phys. 2008104064513.

[27] P. Perlin, T. Suski, H. Teisseyre, M. Leszczynski, I. Grzegory, J. Jun, S. Porowski, P. Bogusławski, J. Bernholc, J. C. Chervin, A. Polian, and T. D. Moustakas "Towards the identification of the dominant donor in GaN" Phys. Rev. Lett., 1995 75, 296.

[28] https://www.nextnano.de/

[29] A. Twitchett, R. Dunin-Borkowski, and P. Midgley, "Quantitative electron holography of biased semiconductor devices” Phys. Rev. Lett., 2002 88, 238302.

[30] D. Iida, K. Tamura, M. Iwaya, S. Kamiyama, H. Amano, and I. Akasaki, "Compensation effect of Mgdoped a-and c-plane GaN films grown by metalorganic vapor phase epitaxy." Journal of Crystal Growth, $2010312,3131-3135$.

[31] W.Li, K. Nomoto, K. Lee, S. M. Islam, Z. Hu, M. Zhu, X.Gao, J. Xie, M. Pilla, D. Jena, and H.Xing "Activation of buried p-GaN in MOCVD regrown vertical structures" Appl. Phys. Lett. 2018 113, 062105

[32] Y. Kuwano, M. Kaga, T. Morita, K. Yamashita, K. Yagi, M. Iwaya, T. Takeuchi, S. Kamiyama, and I. Akasaki, "Lateral Hydrogen Diffusion at p-GaN Layers in Nitride-Based Light Emitting Diodes with Tunnel Junctions” Jpn. J. Appl. Phys. 2013 52, 08JK12

[33] H. Amano, M. Kito, K. Hiramatsu, and I. Akasaki Jpn. "P-Type Conduction in Mg-Doped GaN Treated with Low-Energy Electron Beam Irradiation (LEEBI)” J. Appl. Phys. 28 (1989) L2112.

[34] O. Gelhausen, M. R. Phillips, E. M. Goldys, T. Paskova, B. Monemar, M. Strassburg, and A. Hoffmann "Dissociation of H-related defect complexes in Mg-doped GaN. " 2004 Phys. Rev. B 69, 125210

[35] L. Amichi, I. Mouton, V. Boureau, E. Di Russo, P. Vennéguès, P. De Mierry, A. Grenier, P-H. Jouneau, C. Bougerol and David Cooper "Correlative investigation of Mg doping in GaN layers grown at different 
temperatures by atom probe tomography and off-axis electron holography." Nanotechnology 2019 31.4, 045702. 


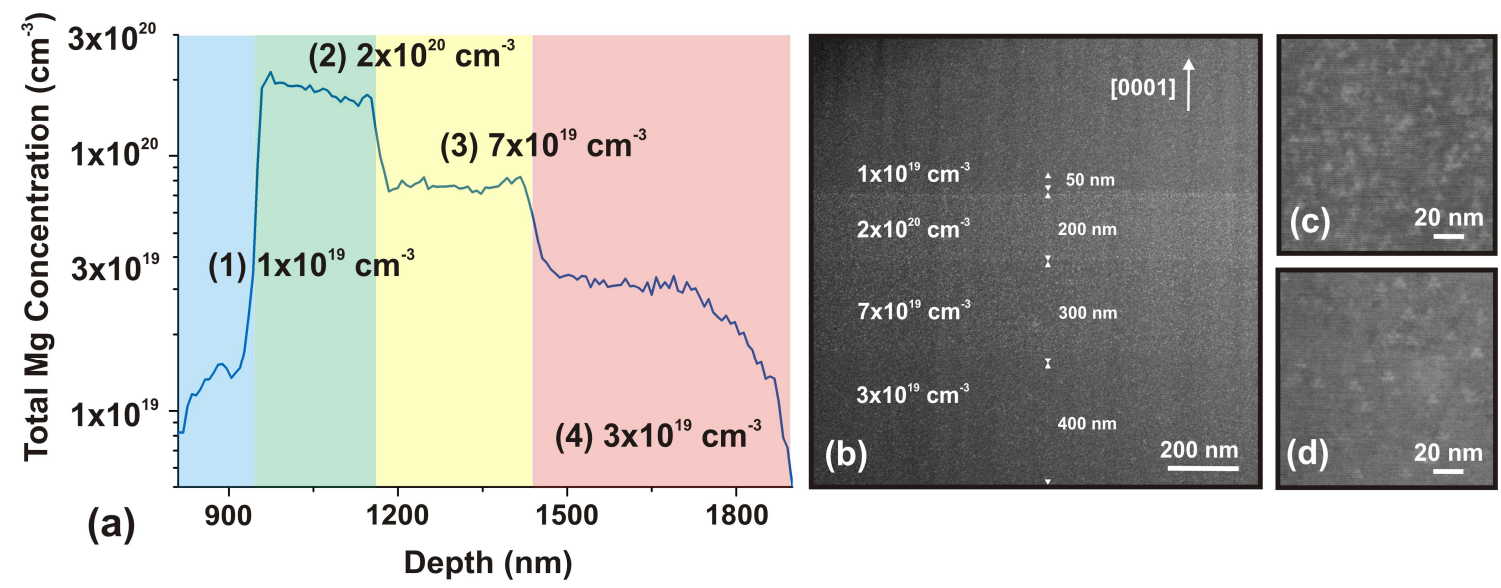

Figure 1 (a) SIMS profile of the Mg dopant. (b) HAADF-STEM image of a cross-section containing the doped layers. Higher magnification images of layers doped at (c) $2 \times 10^{20} \mathrm{~cm}^{-3}$ and (d) $7 \times 10^{19} \mathrm{~cm}^{-3}$ respectively. 


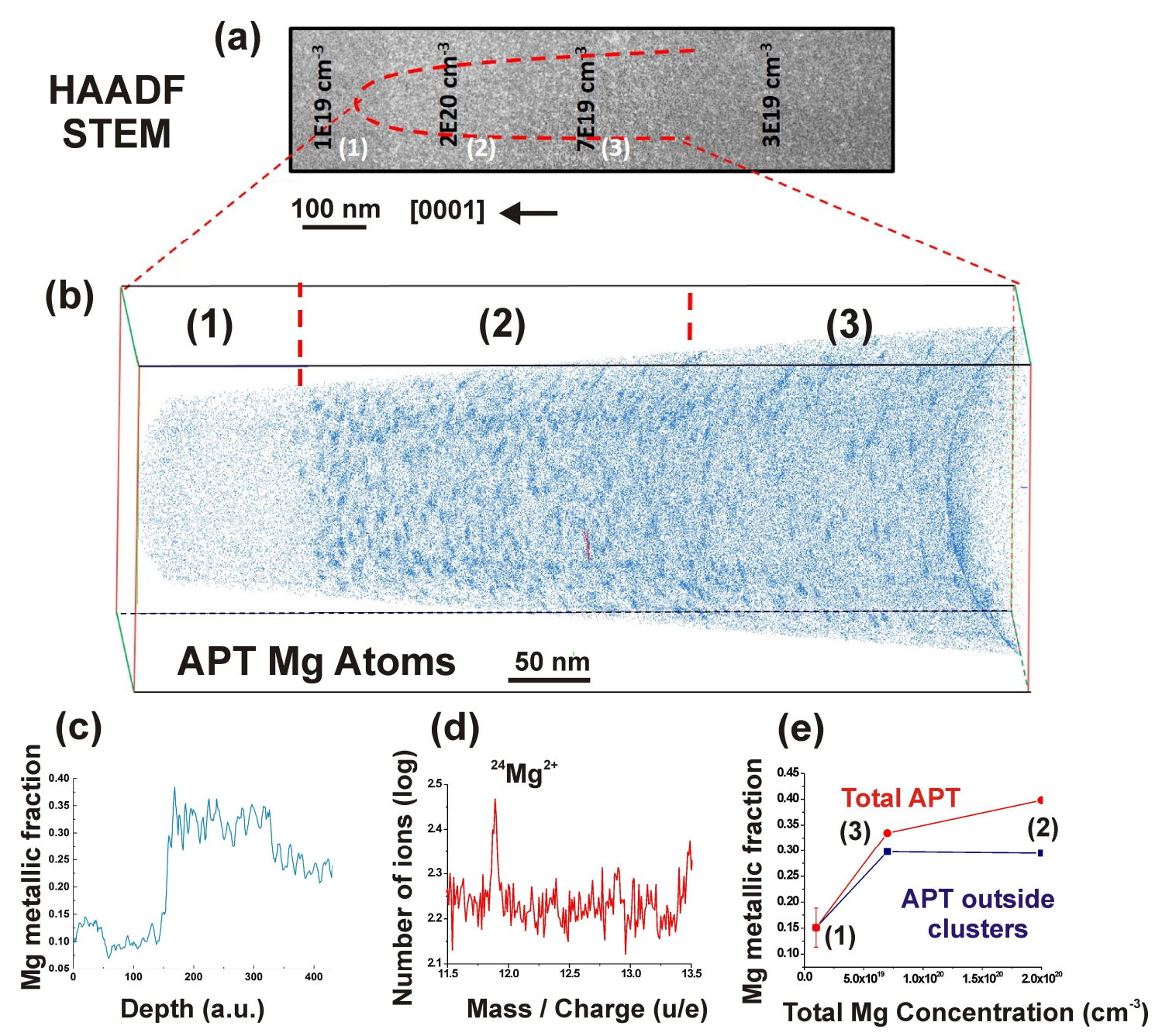

Figure 2 (a) HAADF-STEM image of the successive doped layers. The red dashes are an illustration of where the APT volume can be located. (b) APT 3D reconstruction of $\mathrm{Mg}$ atoms in blue showing three different regions. (c) Metallic site fraction, $y=M g /(M g+G a)(\%)$, profile drawn as a function of the depth. (d) Mass spectrum of region (3). (e) The total metallic site fraction and metallic site fraction in the different regions measured by ATP plotted as a function of Total Mg concentration measured by SIMS. 

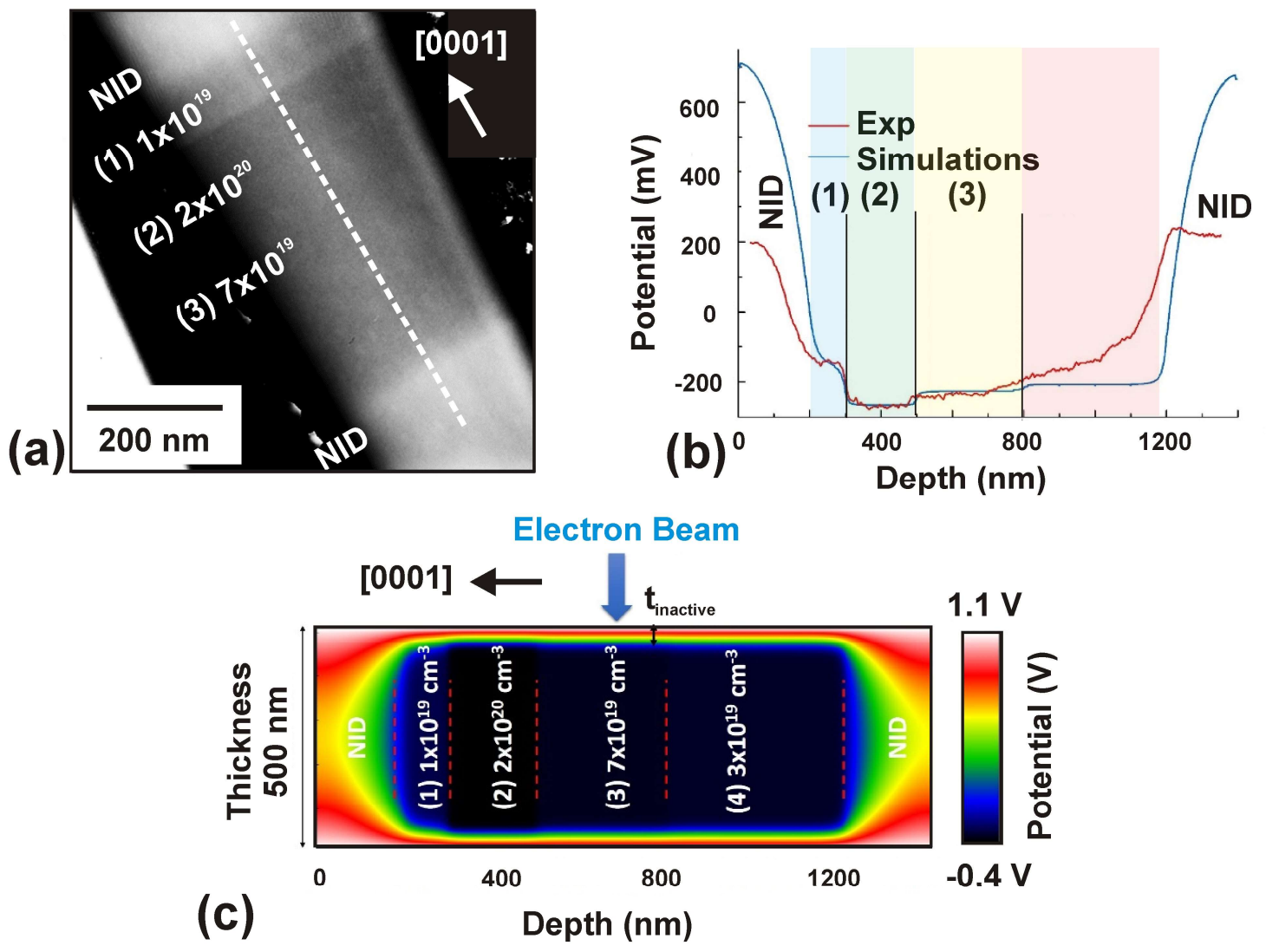

Figure 3(a) Phase image acquired at $400{ }^{\circ} \mathrm{C}$ in-situ heating temperature. (b) Experimental and simulated potential profile as a function of depth in red and blue respectively. (c) Simulation of the 2D distribution of the electrostatic potential in the $500 \mathrm{~nm}$ thick TEM specimen including the inactive thickness. 


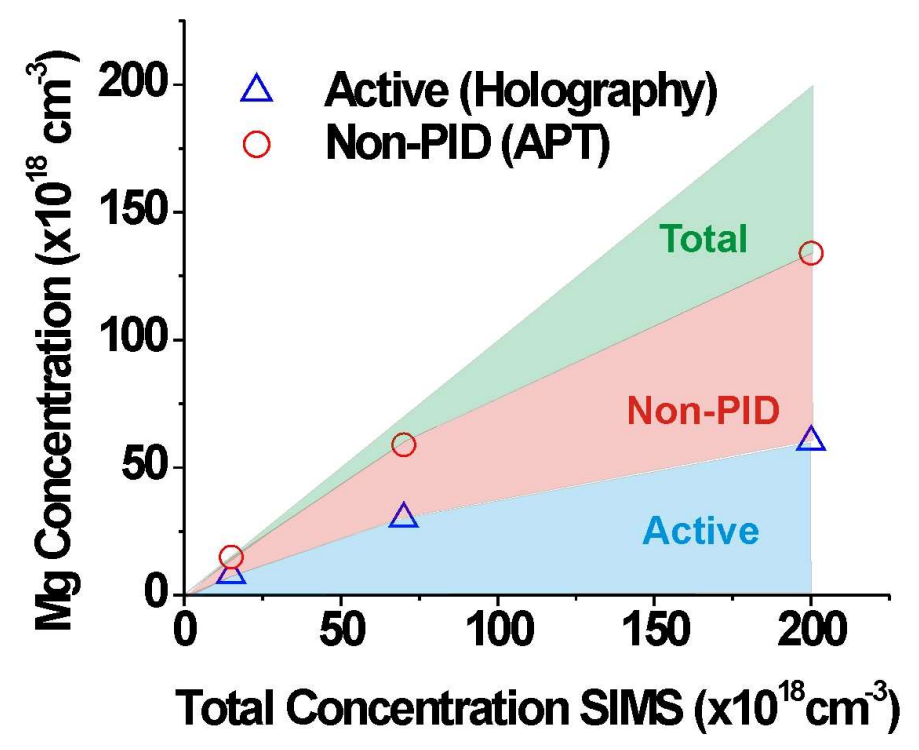

Figure 4. The non-PID (potentially active) Mg measured by APT and the active Mg measured by holography plotted as a function of the total dopant concentration measured by SIMS. The data is taken directly from Table 1. 


\begin{tabular}{|c|c|c|c|}
\hline \multirow{2}{*}{ Region } & \multicolumn{3}{|c|}{ Mg concentration $\left(\times 10^{18} \mathrm{~cm}^{-3}\right)$} \\
\cline { 2 - 4 } & Total (SIMS) & non-PID (APT) & Active (Holo) \\
\hline 1 & 15 & $15\{1.0\}$ & 8 \\
\hline 2 & 200 & $134\{0.67\}$ & 60 \\
\hline 3 & 70 & $59\{0.84\}$ & 30 \\
\hline
\end{tabular}

Table 1 : Shows values of total Mg concentration measured by SIMS. The ratio of the total and non-PID metallic site fractions measured by ATP are shown in \{brackets\}. Quantitative values of the non-PID (potentially active) concentrations are obtained by multiplying the SIMS measurement and the metallic fraction ratio. Finally the active dopant concentration used to fit the simulated potential profiles to the holography results are shown. 